\title{
投弹问题及毁伤价值场图象解法 *
}

\author{
陈 循 \\ (中国科学院系统科学研究所, 北京 100080)
}

\section{关键词覆盖问题、计算机图象、NP 问题、非线性最优化}

所谓设弹问题是这样一个问题: 设有 $N$ 枚威力和投掷精度不尽相同的炸弹, 用来攻击敌 国某区域中的 $M$ 个价值和坚固程度不尽相同的目标, 欲求如何配置这 $N$ 枚炸弹的瞄准点, 使 得对这 $M$ 个目标的打击效果达到极大.

这是一个最优覆盖问题, 具有 NP 难度. 在实际中可以转化为无约束多变最量非线性优 化问题, 用逼近算法求解. 关键在于选用何种模型, 以及如何选择更好的初始化点.

这方面的研究主要集中于美国 $A D$ 报告之中, 其中 Boy ${ }^{[1]}$ 的报告基本覆盖了这方面的 研究. 文献提出用 Powell 共轭方向法求极值, 并提出四种确定初始化点的方案: (1) $N$ 个最 坚固目标的坐标点; (2) $m$ 个价值最大的目标的坐标点; (3) 目标区的重心点; (4) 目标平面 上 $N$ 个随机分布点. 计算表明, 优化结果与初始化方法有关, 文献建议用方案 (2). 邱成龙、 方开泰等在 “大威力武器瞄准决策” 研究报告中, 根据数论均匀布点原理提出总体布点法、聚 类法和前进法作为初始化方法. 黄文奇 ${ }^{[2]}$ 用与众不同的拟物方法, 将目标视为嵌死在绝对光滑 平面上的具有单位质量的质点, 而将覆盖物看作可以在平面上自由滑动的单位质量圆盘. 这 是一个模拟万有引力系统, 每一个质点都吸引圆盘来覆盖自己, 圆盘则屏蔽它已覆盖的质点 引力, 从而使覆盖问题得到解决.

本文提出的毁伤价值场模型及初始化点方法与上述所有方法均不同, 具有更高的效率和 直观可视性. 其中毁伤价值场的卷积计算法源自计算机图象处理技术, 不仅具有重要的军事 价值, 还可以推广到诸如生产线机器人布局, 工厂及配套设施选址, 国家或区域经济区划等社 会经济问题. 为了得到连续的毁伤价值场, 首先要改进毁伤函数.

\section{1 目 标 毁 伤 函 数}

军事目标受到炸弹等武器攻击时, 将完全或部分地失去其军事或经济价值, 称为毁伤. 传 统的毁伤模型为二值不连续函数, 目标被毁伤的价值为

$$
V(r)= \begin{cases}V_{m}, & r \leqslant R_{w}, \\ 0, r>R_{w},\end{cases}
$$

这里 $0 \leqslant r=\sqrt{(x-\alpha)^{2}+(y-\beta)^{2}}$ 是弹着点 $(x, y)$ 与目标点 $(\alpha, \beta)$ 间的距离, $V_{\mathrm{m}}$ 为受攻目标原有价 值, $R_{w}$ 为武器的毁伤半径.

1992-06-10 收稿, 1993-12-29 收修改稿.

*国家自然科学基金青年基金资助项目. 
模型 (1)有三点不足之处. 一是过于简单, 目标价值是全部被毁或是毫无损伤, 与物理事 实不符; 二是参数 $R_{w}$ 将武器性能和目标易损伤性混为一谈; 三是无法为基于一阶或二阶导数 信息的各种优化称法提供必要的梯度信息, 第三个缺点尤为重要, 它实际上使后面的多弹多 目标化过程难于实施.

现将毁伤模型 (1)作了如下改进:

$$
V(r)=\left\{\begin{array}{l}
V_{m}, r \leqslant R_{m}, \\
\frac{P_{w} K}{r^{2}} V_{m}, r>R_{m},
\end{array}\right.
$$

其中 $P_{w}$ 为武器威力; $K=\left(R_{w}^{0}\right)^{2} ; R_{m}=\sqrt{P_{w}} R_{m}^{0}$ 为武器威力 $P_{w}$ 下的目标毁伤半径; 而 $R_{m}^{0}$ 为目标 的标准毁伤半径, 即当武器威力 $P_{w}=1$ 时彻底摧毁目标的距离, 它是目标易毁伤性的度量.

将毁伤率 $D$ 与目标本身的价值 $V_{m}$ 分离, 即得到毁伤函数:

$$
D=\left\{\begin{array}{l}
1, r \leqslant R_{m}, \\
\frac{P_{w} K}{r^{2}}, r>R_{m},
\end{array}\right.
$$

实际上毁伤函数 $D$ 是武器威力 $P_{w}$, 目标易损性 $R_{m}^{0}$ 及弹标距离 $r$ 的函数:

而毁伤价值为 $V=V_{m} D$.

$$
D=D\left(P_{w}, R_{m}^{0}, r\right) \text {, }
$$

在改进模型中武器性能得以与目标性能相分离; 在 $r=0, R_{m}$ 点重新定义后, 函数在整个 定义域连续且有两阶以上的导函数; 目标被毁伤的效果随弹着点与目标的距离平方递减, 符 合下述物理事实: 大威力武器若以冲击波造成的超压为主要摧毁机制, 则这种超压恰与随半 径平方增长的三维球面波波前的表面积成反比.

\section{2 单弹多目标问题及价值场图象}

用一发大威力武器攻击含有 $M$ 个目标的目标群的优化问题, 是多弹多标问题的基础, 单
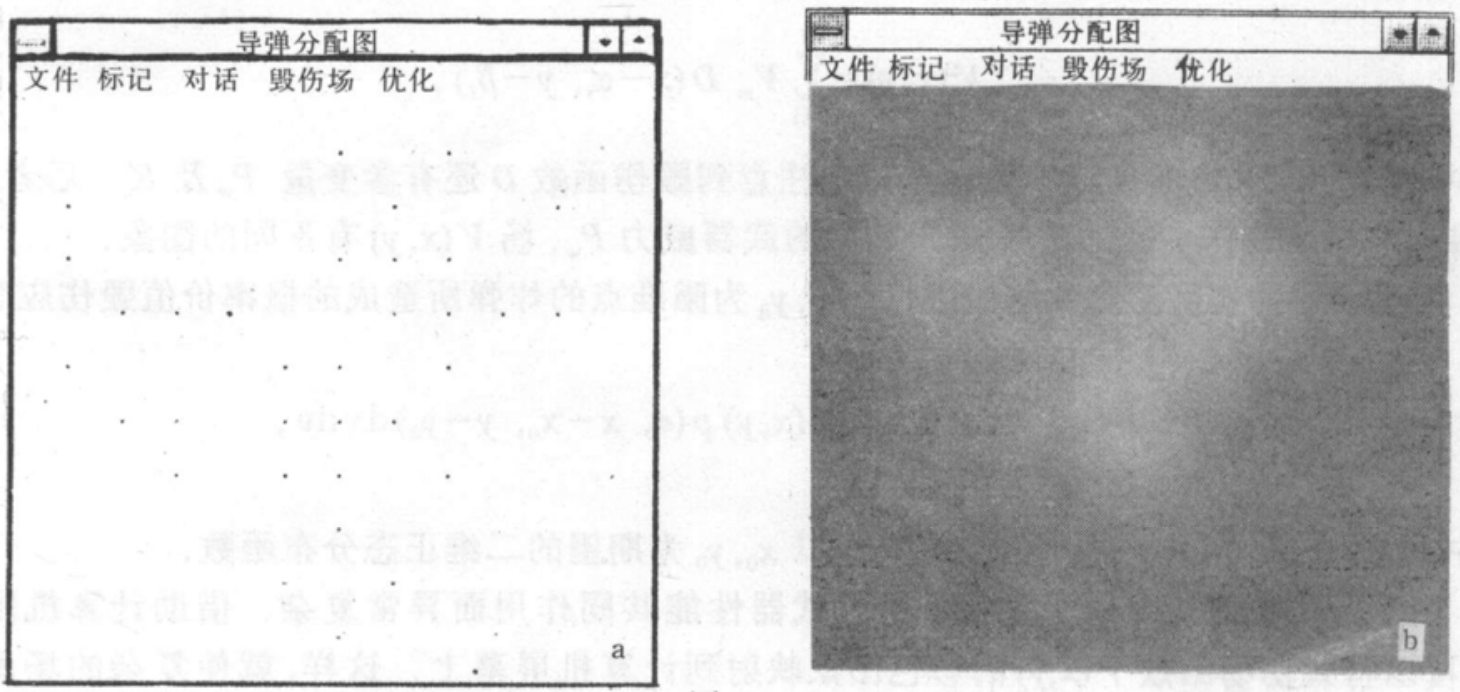

图 1

a. 41 个坚固程度相同、价值相同各为 100 的目标构成的目标群; b. 相应的毁伤价值场图象, 图中三个青蓝 色的区域为极大值域, 图象由 Windows 环境下的 MAG 应用程序生成 
弹瞄准的优化结果, 可以作为多弹多标瞄准优化的初始点.

当目标的受攻平面上分布的密集程度与其自身的易毁伤性及武器的威力相匹配时,一发炸 弹就有可能同时击毁两个以上的目标. 当然, 这与炸弹的实际落点密切相关. 实际上, 目标 平面上的任何一点落下大威力炸弹, 都会造成一定价值的毁伤. 因此, 存在二维毁伤价值标 量场, 用这个场可以精确地描写武器落点与它所造成的目标毁伤程度之间的关系, 称为毁伤 价值场. 毁伤价值场的分布取决于目标群的分布图案、目标的性质以及武器的性能.

为了表示离散目标群原有价值的空间分布图案, 目标原有价值冲量场函数:

$$
V_{m}(x, y)=\left\{\begin{array}{l}
V_{m_{i}}, x=x_{i}, y=y_{i}, \\
0, x \neq x_{i}, \text { 或 } y \neq y_{i},
\end{array} \quad i=1,2, \cdots, M,\right.
$$

其中 $V_{m_{i}}$ 为第 $i$ 个目标的价值, $x_{i}, y_{i}$ 为目标 $i$ 的坐标.

事实上, 毁伤价值场恰为毁伤函数 $D(x, y)$ 与原有价值场 $V_{m}(x, y)$ 的卷积:

$$
V(x, y)=V_{m}(x, y) * D(x, y)=\iint_{-\infty}^{\infty} V_{m}(\alpha, \beta) D(x-\alpha, y-\beta) \mathrm{d} \alpha \mathrm{d} \beta,
$$

只要注意到式 (4) 中 $r=\sqrt{(x-\alpha)^{2}+(y-\beta)^{2}}$. 这不但适用于式 (5)一类的离散型目标分布, 还 可以推广到更为实际的价值连续分布的目标场, 诸如机场跑道, 军事防线, 城市建筑群等. 在 计算机上实现卷积 (6),须采用离散化形式:

$$
V(x, y)=V_{m}(x, y) * D(x, y)=\sum_{i=0}^{I-1} \sum_{j=0}^{J-1} V_{m}(i, j) D(x-i, y-j),
$$

在这里用 $x=1,2, \cdots, I-1$ 及 $y=1,2, \cdots, J-1$ 处的值描述卷积 $V_{m}(x, y) * D(x, y)$ 的一个大小为 $I \times J$ 的周期阵列. 当然, 此处的卷积因子 $V_{m}(x, y)$ 及 $D(x, y)$ 需作周期性延拓.

对于象式 (5) 那样的离散目标价值场, 除去目标所在点之外场值均为零, 卷积 (7) 退化为更 简单的形式:

$$
V(x, y)=\sum_{i=1}^{M} V_{m_{i}} D\left(x-\alpha_{i}, y-\beta_{i}\right),
$$

其中 $\alpha_{i}, \beta_{i}$ 为目标 $i$ 的坐标. 在计算中应注意到毁伤函数 $D$ 还有参变量 $P_{w}$ 及 $R_{m_{i}}^{0}$, 后者为第 $i$ 个目标的标准毁伤半径. 显然,对于不同的武器威力 $P_{w}$, 场 $V(x, y)$ 有不同的图象.

考虑到导弹实际落点的随机性, 以 $x_{0}, y_{0}$ 为瞄准点的炸弹所造成的概率价值毁伤应为:

$$
V\left(x_{0}, y_{0}\right)=\int_{-\infty}^{\infty} \int_{-\infty}^{\infty} V(x, y) p\left(\sigma, x-x_{0}, y-y_{0}\right) \mathrm{d} x \mathrm{~d} y,
$$

其中 $p\left(\sigma, x-x_{0}, y-y_{0}\right)$ 是标准差为 $\sigma$, 以 $x_{0}, y_{0}$ 为期望的二维正态分布函数.

由于毁伤价值场 $V(x, y)$ 受目标群及武器性能共同作用而异常复杂. 借助计算机图象技 术, 可以将毁伤场函数 $V(x, y)$ 的彩色图象映射到计算机屏幕上. 这样, 就使复杂的场函数变 得直观形象, 可以帮助, 甚至启发决策者, 特别是高级军事指挥官迅速选定最佳投弹瞄准点. 对于单枚导弹而言，毁伤价值场中价值最大的一点就是全局最佳瞄准点. 
人类完善的右脑图象识别机制可以毫不费力地找到 $V(x, y)$ 图象的最佳瞄准域, 但不够精 确. 为了满足实用的精度要求, 我们提出了圆周搜索法, 这是一个基于计算机图象的极大值 逼近算法. 其基本思想极为简单: 在价值场平面上先以目视选一初始点 $\left(x_{0}, y_{0}\right)$ 为圆心, 取 $R=3.03 \sigma$ 为半径作圆周, 在圆周上检测每一象素的颜色 (即价值), 分别按四个象限求和:

$$
V_{k}=\sum_{i} V\left(x_{k_{i}}, y_{k_{i}}\right), k=1,2,3,4,
$$

其中 $x_{k_{i}}, y_{k_{i}}$ 为圆周象限 $k$ 上离散点列的坐标. 最后, 比较四个圆周象限上的价值和 $V_{k}$, 并使圆 心 $\left(x_{0}, y_{0}\right)$ 向价值和较大的方向移动一个象素到 $\left(x_{1}, y_{1}\right)$, 如此循环, 直到圆周上各方向上的价值 均衡为止.

这里 $\sigma$ 为武器随机落点正态分布函数的均方差, $3.03 \sigma$ 是百分之九十九的弹着命中圆半 径. 显然, 搜索的结果与选用的武器投掷精度及初始点有关. 它可以协同人眼对色彩辨识的 主观感受, 迅速定位最佳单弹瞄准点.

圆周搜索法与经典的梯度算法不同, 由于它对函数的测试不局限在一个很小的邻域, 而是 跨越一个足够大的圆域 $R$, 所以圆周搜索法有跨越局部极值点和鞍点的能力, 其跨越能力与 测试圆直径有关.

\section{3 多弹多目标问题}

当使用多枚具有同种爆炸威力的炸弹攻击群目标时, 若毁伤价值场出现的多个极大值域 相距较远, 以至足以避免投向这几个极大值点的炸弹重复毁伤某些目标, 那么, 重复使用单弹 瞄准的场图象法就可以求解多弹多目标问题. 这时, 只要分别对这些分离的极值点分配一枚 炸弹就是了. 但实际问题中, 重复毁伤是无法避免的. 这种情况下, 单发弹优化的简单迭加 的结果并非是多弹瞄准的全局最优解. 其结果值因没有扣除已被其他炸弹毁伤的价值而偏 高. 实际上, 多弹问题的描述类似于随机事件的联合发生的概率问题.

设有 $N$ 枚具有威力 $P_{w_{1}}$ 和命中精度 $\sigma_{i}$ 的炸弹 $i=1,2, \cdots, N$, 及 $M$ 个具有价值 $V_{j}^{0}$ 和毁伤 半径 $R_{m_{j}}^{0}$ 的目标 $j=1,2, \cdots, M$, 则当炸弹 $i$ 瞄准目标平面上的 $\left(x_{i}, y_{i}\right)$ 时, 它对目标 $j$ 的概率毁 伤为

$$
D_{i j}^{p}=\int_{-\infty}^{\infty} \int_{-\infty}^{\infty} D_{i j} p_{i} \mathrm{~d} x \mathrm{~d} y
$$

其中

$$
D_{i j}=D\left(P_{w_{i}}, R_{m_{j}}^{0}, \alpha_{j}, \beta_{j}, x, y\right)=\left\{\begin{array}{l}
1, \quad d_{j} \leqslant R_{m_{j}}, \\
\frac{P_{w_{i}}\left(R_{m_{j}}^{0}\right)^{2}}{d_{j}^{2}}, d_{j}>R_{m_{j}},
\end{array}\right.
$$

$d_{j}=\sqrt{\left(x-\alpha_{j}\right)^{2}+\left(y-\beta_{j}\right)^{2}}, R_{m_{j}}=\sqrt{P_{\mathrm{w}_{1}}} R_{m_{j}}^{0}$, 而其中 $p_{i}$ 为服从正态分布的概率.

$$
p_{i}=p\left(\sigma_{i}, x_{i}, y_{i}, x, y\right)=\frac{1}{2 \pi \sigma_{i}^{2}} \mathrm{e}^{-\frac{\left(x-x_{i}\right)^{2}+\left(y-y_{i}\right)^{2}}{2 \sigma_{i}^{2}}} .
$$

$N$ 枚弹对目标 $j$ 的合并毁伤为 


$$
V_{j}=V_{j}^{0}\left[1-\prod_{i}^{N}\left(1-D_{i j}^{p}\right)\right],
$$

$N$ 枚弹对 $M$ 个目标的总毁伤为

$$
V=\sum_{j}^{M} V_{j}=\sum_{j}^{M} V_{j}^{0}\left[1-\prod_{i}^{N}\left(1-D_{i j}^{p}\right)\right] .
$$

多弹多目标瞄准的优化决策问题即求解下式的最大化:

$$
\max V=\max _{x_{i}, y_{i}} \sum_{j}^{M} V_{j}^{0}\left[1-\prod_{i}^{N}\left(1-D_{i j}^{p}\right)\right],
$$

其中 $x_{i}=x_{1}, x_{2}, \cdots, x_{N} ; y_{i}=y_{1}, y_{2}, \cdots, y_{N}$, 为 $N$ 枚弹分别瞄准的坐标.

求解式 (15) 实际上是一个 $2 N$ 维无约束非线性规划问题, 可以采用任何一种多变量极大化 的解法. 众所周知, 关键在于 $N$ 个初始瞄准点的选定. 初始瞄准点的设定是组合优化问题, 其计算复杂性为选排列数

$$
A_{n}^{k}=\frac{n !}{(n-k) !},
$$

式中 $n$ 为拟用弹数, $k$ 为目标数或目标分组数. 例如对于有一个有 41 个目标和 3 枚导弹的 问题, 若以单个目标为初始瞄准点 $(k=41)$, 则布点的可能性有 63960 种.

按照本文的主张, 应把初始瞄准点定位在目标价值场中的极值点上 $(k=3$, 参考图 1), 所 用弹数与瞄准点数对应 $(n=3)$, 这样初始布点的可选方案数减少到 6 种, 万分之一! 如果考 虑使用不同威力的核弹, 则应按弹的威力大小, 把它们依次按排在价值场中极值域面积或价 值由大到小的各对应极值点上, 因而初始点的配置方案只有一种. 此外, 由于作为核心的卷 积计算可以用十分成熟的硬件芯片代劳, 从而使本方法完全可以实时进行, 这无疑具有重要 的军事价值. 比如用作轰炸机的瞄准辅助图象系统.

实算表明,利用毁伤价值场的图象的极值点定位投弹问题的初始瞄准点, 比文献中的定点 方法更具理论合理性, 不但收玫迅速, 能得到全局最优解, 而且直观明确.

\section{参考文献}

[1] Boy, E. G., AD-A141034, 1984.

[ 2] 黄文奇,计算机学报, $1989, \mathbf{5}: 610-616$. 